\title{
Combined Endoscopic Endonasal Transorbital Approach with Transconjunctival-Medial Orbitotomy for Excisional Biopsy of the Optic Nerve: Technical Note
}

\author{
Maria Koutourousiou ${ }^{1}$ Paul A. Gardner ${ }^{1}$ S. Tonya Stefko ${ }^{2}$ Alessandro Paluzzi ${ }^{1}$ \\ Juan C. Fernandez-Miranda ${ }^{1}$ Carl H. Snyderman ${ }^{3}$ Joseph C. Maroon ${ }^{1}$ \\ ${ }^{1}$ Department of Neurological Surgery, University of Pittsburgh School \\ of Medicine, Pittsburgh, Pennsylvania, United States \\ 2 Departments of Ophthalmology, University of Pittsburgh School of \\ Medicine, Pittsburgh, Pennsylvania, United States \\ ${ }^{3}$ Department of Neurological Surgery and Department of

\begin{abstract}
Address for correspondence and reprint requests Paul A. Gardner, M.D., Department of Neurological Surgery, University of Pittsburgh School of Medicine, 200 Lothrop Street, Suite B400, Pittsburgh, PA, USA
\end{abstract} \\ 15213 (e-mail: gardpa@upmc.edu).
} Otolaryngology, University of Pittsburgh School of Medicine,

Pittsburgh, Pennsylvania, United States

J Neurol Surg Rep 2012;73:52-56.

\begin{abstract}
Background Access to the intraorbital optic nerve segment can be facilitated via a transcranial approach that allows access to the entire orbital cavity. The endoscopic endonasal approach (EEA) combined with a transconjunctival-medial orbitotomy represents an alternative technique to achieve the same goal.

Objective Report a surgical technique that allows total resection of the intraorbital optic nerve with minimal trauma and excellent results. Further extend and define the limits and indications of the EEA to orbital surgery.

Methods A patient with rapidly progressive, but asymmetric, vision loss underwent EEA for optic nerve biopsy. Due to the undetermined histopathological diagnosis and complete unilateral vision loss, diagnostic total optic nerve resection was indicated. The entire intraorbital length of the nerve was resected via an endoscopic endonasal

\section{Keywords}

- endoscopic endonasal approach

- optic nerve resection

- orbital surgery

- transconjunctivalmedial orbitotomy transorbital approach combined with transconjunctival-medial orbitotomy.

Results A 2-cm intraorbital nerve segment was sent for pathological examination. The patient maintained normal extraocular movements and experienced no complications. The postoperative course was uneventful and the patient was discharged the next day. Conclusion The EEA provides another option for access to the entire optic nerve. It is a safe and effective technique lacking cosmetic defects and providing an alternative corridor to traditional transcranial approaches to the orbit.
\end{abstract}

\section{Introduction}

The fronto-orbito-zygomatic (FOZ) approach is traditionally employed for access to the orbital cavity, exposure of the orbital apex, and removal of the optic nerve. ${ }^{1,2}$ Alternatively, lateral orbitotomy allows access to the retrobulbar space; it cannot, however, provide exposure of the optic canal. ${ }^{2}$ The endoscopic endonasal transorbital approach can facilitate a medial access to the entire retrobulbar space through the medial orbital wall. Posterior extension of the exposure to the orbital apex and optic canal can reveal the entire canalicular received

November 7, 2011

accepted after revision

May 1, 2012

published online

August 18, 2012
Copyright $\odot 2012$ by Thieme Medical Publishers, Inc., 333 Seventh Avenue, New York, NY 10001, USA. Tel: +1(212) 584-4662.
DOI http://dx.doi.org/ 10.1055/s-0032-1323156. ISSN 2193-6358. 


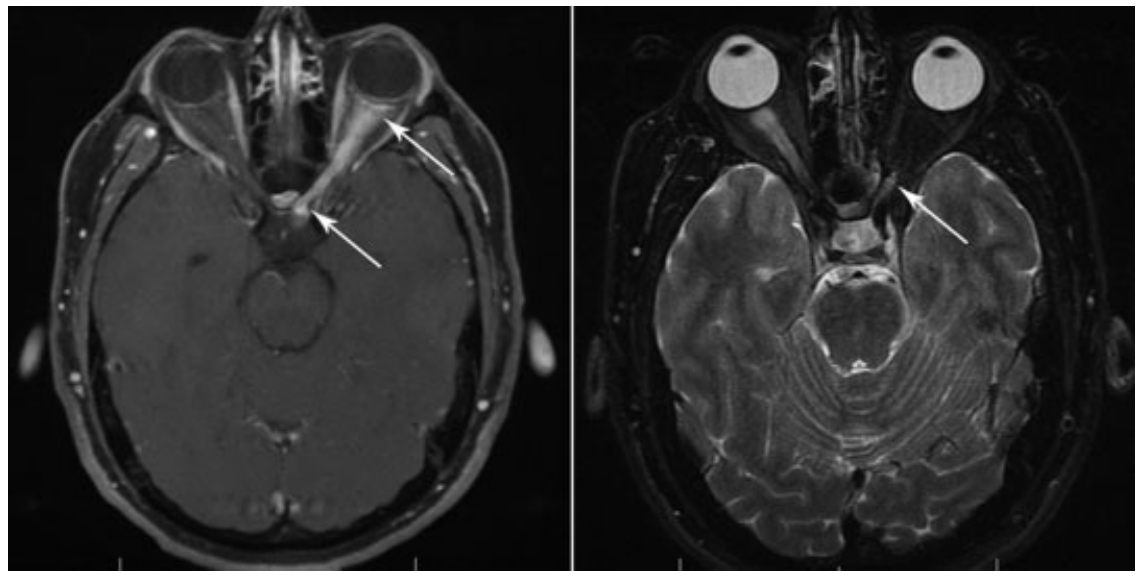

Figure 1 Left: Preoperative axial T1-weighted magnetic resonance imaging (MRI) shows an enlarged left optic nerve that enhances after contrast administration. The pathological signal of the nerve extends from its global attachment all the way back to its intracranial segment (arrows). Right: Postoperative axial T2-weighted MRI demonstrates the resection of the intraorbital left optic nerve up to the level of the annulus of Zinn (arrow) and the intact orbital anatomy with symmetric and aligned eye globes.

optic nerve. Further removal of the tuberculum sellae and medial opticocarotid recess ( $\mathrm{mOCR}$ ) provides access to the intracranial optic nerve back to the optic chiasm. Combining this technique with the inferomedial transconjunctival approach (medial microorbitotomy) to gain access and transect the optic nerve at its attachment to the globe, resection of the intraorbital optic nerve can be achieved in its entirety.

\section{Illustrative Case}

A 35-year-old male experienced rapidly progressive vision loss (OS 2/10) and pain with left eye movements. For a presumed diagnosis of optic neuritis, the patient was treated with steroids and his pain improved. However, despite steroid therapy, the left eye progressed to no light perception, and the right eye began to be affected. Detailed laboratory examinations were all within normal limits. Magnetic resonance imaging (MRI) revealed an enlarged left optic nerve with pathologic brisk enhancement that extended from the posterior margins of the globe to the left aspect of the chiasm (-Fig. 1 left). The patient underwent the endoscopic endonasal approach (EEA) for optic nerve decompression and biopsy of the tumor. The pathology was not diagnostic, describing patchy lymphohistiocytic inflammatory infiltrate within the nerve with near complete axon loss.

Following surgery, the patient continued to lose vision in the functioning right eye. A second-stage EEA was offered for total resection of the affected left optic nerve to obtain a larger specimen for pathological diagnosis and attempt to prevent disease extension to the other eye.

The final pathological study revealed a noninfectious inflammatory lesion of uncertain etiology, excluding glial tumor, lymphoma, or demyelinating condition. Unfortunately for the young patient, vision of the functional eye continued to deteriorate after surgery.

\section{Surgical Technique}

(see $\boldsymbol{- V i d e o} 1$ online.)
Although the procedure took place in two stages (stage 1: sphenoethmoidectomy, medial orbital decompression and optic nerve decompression; stage 2: transconjunctival transorbital approach and endoscopic transnasal approach), we describe the whole technique as one operation.

\section{Sphenoethmoidectomy}

The nasal cavity was inspected with a 0-degree endoscope. The posterior nasal septum was incised and disarticulated from the rostrum of the sphenoid bone. The bone of the rostrum was removed, creating an opening into the sphenoid sinus bilaterally; this opening was maximally enlarged to the margins of the sinus. The inferior aspect of the left middle turbinate was transected and removed. The uncinate process was removed, creating an opening into the maxillary sinus; the maxillary antrostomy was further enlarged posteriorly and superiorly. The ethmoid air cells were then removed in an anterior-to-posterior direction up to the level of the anterior ethmoidal artery fully exposing the medial wall of the orbit.

\section{Medial Orbital Decompression and Optic Nerve Decompression}

The lamina papyracea was fractured and elevated from the underlying periorbita from the orbital floor up to the level of the skull base. Removal of the bone continued posteriorly to the orbital apex. The course of the optic nerve in the canal was visualized and confirmed with image guidance. Under

\section{Video 1}

Intraoperative video demonstrating the resection of the optic nerve with combined endoscopic endonasal transorbital approach and inferomedial transconjunctival approach.

Online content including video sequences viewable at:www.thieme-connect.com/ejournals/html/

$10.1055 / \mathrm{s}-0032-1323156$ 
endoscopic visualization, the bone overlying the orbital apex up to the superior orbital fissure was removed to completely expose the canalicular optic nerve. Additional bone of the anterior clinoid, sella, and tuberculum sellae was removed and the lateral and MOCR were exposed. The periorbita was then incised at the apex and the incision continued posteriorly along the sheath of the optic nerve to fully decompress it. The incision continued anteriorly to facilitate decompression of the total length of the medial orbit. The orbital fat was cauterized and reduced with bipolar electrocautery.

\section{Transconjunctival Approach}

The external orbital approach was performed with an incision into the conjunctiva inferomedially to isolate the inferior rectus muscle. The conjunctival incision was extended toward the limbus and an $\sim 75$-degree peritomy was done. A relaxing incision was performed superomedially in the conjunctiva. The medial rectus muscle was isolated and detached from the globe. A 6-0 Vicryl baseball stitch was used to suture through the tendinous attachment so that the globe could be retracted laterally. A malleable retractor was placed medially to hold back the orbital fat, and the globe was further rotated laterally to bring the optic nerve into view. A right-angle clamp was placed around the optic nerve just posterior to the globe and tightened (-Fig. 2). Through the transconjunctival approach, the optic nerve was transected at its attachment to the globe just posterior to the clamp.

\section{Transorbital Approach}

Through the incised periorbita of the medial orbital wall, the medial and inferior rectus muscles were identified, and

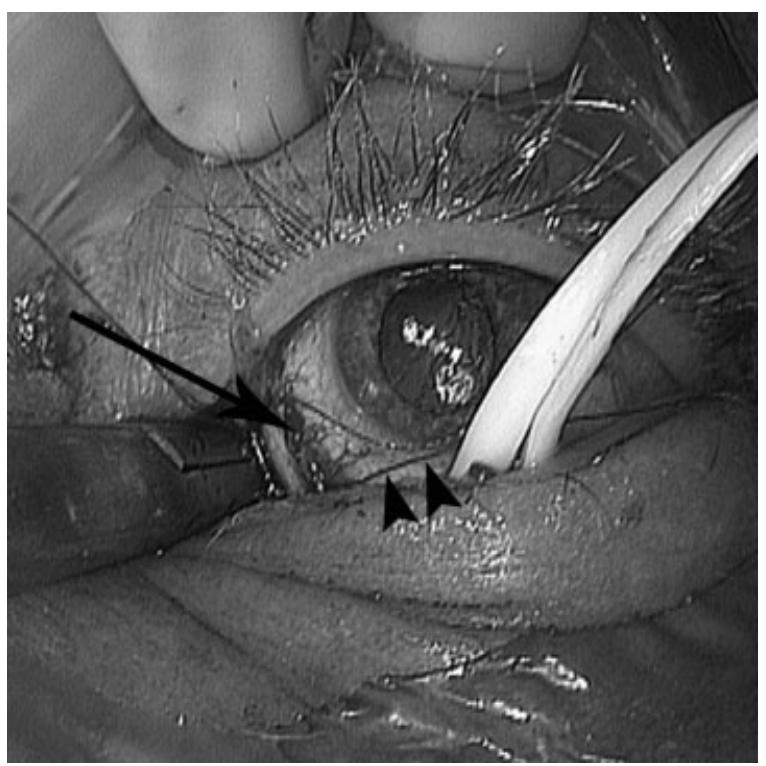

Figure 2 Inferomedial transconjunctival approach. The inferior rectus muscle has been isolated and retracted with the yellow vessel loop. The medial rectus muscle has been detached from the globe (arrow), and a suture (arrowheads) is placed through the tendinous attachment to facilitate lateral rotation of the globe and exposure of the optic nerve. The clamp is placed around the optic nerve at its attachment to the globe. dissection between them was performed. The annulus of Zinn was visualized and transected between these muscles (-Fig. 3). This allowed further release of the muscles and dissection between them to identify the optic nerve at the orbital apex and optic canal and transect it. The nerve was carefully dissected free from the annulus of Zinn. The ophthalmic artery was bipolar coagulated and ligated. Care was taken to avoid manipulations at the lateral aspect of the annulus to prevent injury to the oculomotor nerves. After transconjunctival transection of the optic nerve at its attachment to the globe, the 2-cm intraorbital segment of the optic nerve was subsequently removed en bloc endonasally through the defect of the medial orbital wall ( - Fig. 4).

There were no vascular injuries, and some minor venous bleeding from the annulus of Zinn was controlled with bipolar coagulation. Adequate hemostasis was obtained with copious warm water irrigation. The endonasal defect was covered with fibrin glue. The medial rectus muscle was reattached to its original scleral incision and the conjunctiva was closed. The patient tolerated the procedure well. The postoperative course was uneventful and the patient was discharged the next day. The nasal splint was removed at the first follow-up visit 6 days after surgery. Mild nasal crusting was noticed at the 4-week follow-up, requiring debridement. Otherwise, healing was appropriate and no further nasal complications occurred.

\section{Discussion}

The indications for optic nerve resection are usually associated with the presence of an orbital lesion that invades the nerve. $^{3}$ In our case, the histological diagnosis was not

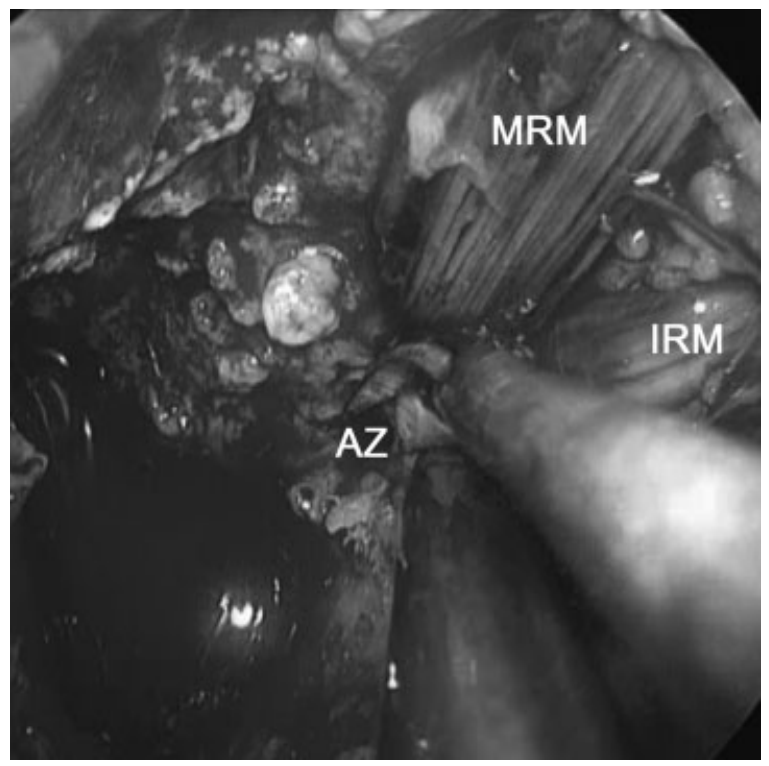

Figure 3 Intraoperative image with a 0-degree endoscope. The bone over the orbital apex and the medial wall of the left orbit has been removed and the periorbita has been incised revealing the medial rectus muscle (MRM), the inferior rectus muscle (IRM) and at the level of the orbital apex the annulus of Zinn (AZ). The annulus is transected with scissors between these muscles avoiding potential injuries of the $\mathrm{CN}$ III, IV, and VI and the branches of the ophthalmic division of CN V, which lie at the lateral aspect of the annulus. 


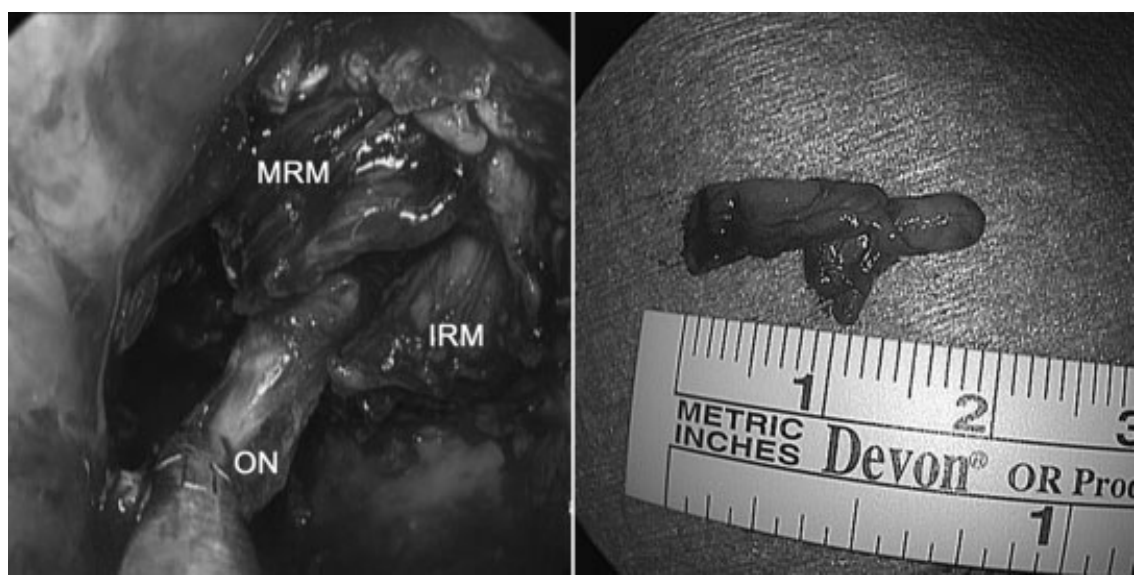

Figure 4 Left: Intraoperative image with a 0-degree endoscope while removing the optic nerve (ON) between the medial rectus muscle (MRM) and the inferior rectus muscle (IRM). Right: The surgical specimen, the 2-cm intraorbital segment of the optic nerve, after being removed en bloc through the endoscopic endonasal approach.

conclusive after biopsy, and complete sectioning of the nonfunctioning nerve was considered for final pathological diagnosis and attempted prevention of lesion extension.

The best surgical approach to the orbit is guided by the location of the lesion ${ }^{4}$ and is designed to give direct access to the optic nerve minimizing trauma to adjacent tissues with good cosmetic result. The traditional surgical approaches to the orbit have been broadly grouped into anterior, lateral, and superior ${ }^{2,3}$ :

1. Anterior orbitotomy is used for lesions in the anterior orbital space. The approach cannot provide access deep in the apex; thus, it is not sufficient for total optic nerve resection. ${ }^{5,6}$

2. Lateral orbitotomy allows access to the retrobulbar space; it involves a lateral and sub-brow skin incision with partial removal of the lateral orbital wall. This approach is contraindicated for resection of pathologies that extend into the optic canal ${ }^{2}$ and has the potential complication of lateral rectus muscle paresis, upper eyelid ptosis, and orbital hemorrhage. $^{7}$

3. The superior approaches provide a panoramic view of the orbit, the orbital apex, the optic canal, and the adjacent intracranial structures. ${ }^{1,2} \mathrm{~A}$ frontal or frontotemporal craniotomy combined with an orbitozygomatic craniotomy is used for tumors with intracranial extension and for those located in the orbital apex and deep medial orbital compartment.

With the goal of resection of the whole intraorbital optic nerve, the best open approach that could provide a panoramic view and facilitate access from the globe to the orbital apex would be the FOZ. ${ }^{1,2,5}$ To achieve an adequate and safe exposure to the orbital apex, a bicoronal skin incision and extensive bone removal are required. Following the superior orbitotomy, the exposure of the superior orbital fissure and the unroofing of the optic canal, the periorbita is opened and the annulus of Zinn must be incised apically to mobilize that portion of the nerve. This maneuver carries the risk of damaging cranial nerve (CN) III, IV, and VI, and branches of the ophthalmic division of CN V. CN IV, which lies directly under the periorbita, is very difficult to spare even with microdissecting techniques during manipulations to the annulus. $^{2}$

We employed a combined endoscopic endonasal transorbital approach with a transconjunctival-medial orbitotomy. The approach requires the joint efforts of endoscopic skull base surgeons (neurosurgeon, sinonasal surgeon) and orbital surgeon (oculoplastic surgeon or neuro-ophthalmologist). This approach achieves direct exposure of the inferior and medial aspects of the orbit and orbital apex without the need of extended craniotomies and potential manipulations on the intraorbital cranial nerves. ${ }^{8}$ During this approach, the potential hemorrhage from the ethmoidal arteries is prevented by indentifying and coagulating them. These arteries provide the landmarks to enter the orbital cavity: the anterior ethmoidal artery lies at the level of the posterior surface of the globe defining the attachment point of the optic nerve to the globe; the posterior ethmoidal artery lies 4 to $7 \mathrm{~mm}$ anterior to the optic canal. Following the removal of the medial wall of the orbit and the incision of the periorbita, the optic nerve is accessed between the medial and inferior rectus muscles. These muscles are mobilized on vessel loops, both to identify them and to create adequate working space. The medial transconjunctival approach is necessary not only for the retrobulbar transsection of the optic nerve but also for the detachment of the medial rectus muscle from the globe to better mobilize it. With the endoscopic endonasal approach, the annulus of Zinn is transected medially between the medial and the inferior rectus muscles, exposing the patient to minimal danger of injury to CN III, IV, VI, and V1.

The endoscopic endonasal transorbital approach demonstrates access to the optic nerve that could serve several indications such as complete resection of tumors involving the nerve such as gliomas and optic nerve sheath meningiomas with pre-existing complete vision loss; partial removal and debulking of tumors such as large optic nerve sheath meningiomas, schwannomas of associated nerves along the tract of the optic nerve, and tumors involving the medial and 
inferior orbital musculature; management of trauma cases with the goal of vision preservation or recovery. When the endoscopic endonasal transorbital approach is combined with a transconjunctival-medial orbitotomy, it can facilitate access to the entire intraorbital segment of the optic nerve. A transcaruncular approach, a potential alternative, is less desirable for this procedure because of the need for direct access to the retrobulbar optic nerve. A transcaruncular route would involve moving aside the orbital fat and medial rectus muscle, whereas dividing the muscle at its tendinous insertion and retracting medially provides direct access to the intraconal retrobulbar space.

There were no permanent complications associated with the technique. The patient was discharged the first postoperative day without any complaints. Extraocular movements were initially decreased (adduction was limited), but recovered. Medial rectus transection and reattachment generally has no long-term impact on vision. Immediate postoperative minimal diplopia is common but clears spontaneously ${ }^{7}$; if diplopia persists, it can be corrected by an oculoplastic surgeon. Ptosis of the upper eyelid, a common complication of orbital surgery, ${ }^{7}$ was not observed in our case. Nasal morbidity is usually minimal and well tolerated in this patient population. ${ }^{9}$ Nasal complications such as sinusitis did not occur. However, nasal crusting, which is common after endonasal surgery, was noticed; endoscopic debridement in an outpatient basis and postoperative nasal care with irrigations are usually adequate to treat this minor complication. There was no cerebrospinal fluid leak and no skin incision or bone removal that could result in cosmetic defects and deformities. At last follow-up, the globes were symmetric and aligned (-Fig. 1 right), bulbar movements were intact, and the nasal cavity was healed appropriately.

The endoscopic endonasal transorbital approach combined with a transconjunctival-medial orbitotomy can be considered the endoscopic equivalent of the traditional FOZ approach with less bone removal, no cutaneous incisions and cosmetic problems, shorter surgical time, faster recovery, and potentially decreased trauma to the orbital structures. It is ideal for access to the medial and inferior optic nerve.

\section{Conclusion}

The development of new endoscopic endonasal techniques can provide an alternative to transcranial incursion into the orbit. The endoscopic endonasal transorbital approach represents a safe and effective choice for excisional biopsy of the optic nerve without some of the complications of the traditional open approaches. A multidisciplinary team with experience in endoscopic endonasal surgery is required for safe execution, but can extend the surgical armamentarium to access the entire optic nerve.

\section{References}

1 Rootman J, Durity FA. Orbital tumors, in Atlas of Neurosurgical Techniques. Brain by Sekhar L. N, Fessler R. G (eds) in Thieme Medical Publishers, Inc., New York, 2006:599-607

2 Maroon JC, Kennerdell JS. Surgical approaches to the orbit. Indications and techniques. J Neurosurg 1984;60:1226-1235

3 Liu GT, Volpe NJ, Galetta SL. Orbital disease in neuro-ophthalmology, in Neuro-Ophthalmology Diagnosis and Management $2^{\text {nd }}$ edition by Elsevier - Health Sciences Division, 2010:612-652

4 Brusati R, Goisis M, Biglioli F, et al. Surgical approaches to cavernous haemangiomas of the orbit. Br J Oral Maxillofac Surg 2007; 45:457-462

5 Bejjani GK, Cockerham KP, Kennerdel JS, Maroon JC. A reappraisal of surgery for orbital tumors. Part I: extraorbital approaches. Neurosurg Focus 2001;10:E2

6 Cockerham KP, Bejjani GK, Kennerdell JS, Maroon JC. Surgery for orbital tumors. Part II: transorbital approaches. Neurosurg Focus 2001;10:E3

7 Dutton JJ. Orbitotomy procedures, in Atlas of Ophthalmic Surgery, Vol II, by Mosby-Year Book, Inc. 1992

8 Kassam A, Snyderman CH, Mintz A, Gardner P, Carrau RL. Expanded endonasal approach: the rostrocaudal axis. Part I. Crista galli to the sella turcica. Neurosurg Focus 2005;19:E3

9 Pant H, Bhatki AM, Snyderman CH, et al. Quality of life following endonasal skull base surgery. Skull Base 2010;20:35-40 